\title{
Calculating ultra-low-frequency wave power of the compressional magnetic field vs. $L$ and time: multi-spacecraft analysis using the Van Allen probes, THEMIS and GOES
}

\author{
Theodore E. Sarris ${ }^{1,2}$ and Xinlin $\mathbf{L i}^{2}$ \\ ${ }^{1}$ Department of Electrical Engineering, Democritus University of Thrace, Xanthi, Greece \\ ${ }^{2}$ Laboratory for Atmospheric and Space Physics, University of Colorado, Boulder, Colorado, USA \\ Correspondence to: Theodore E. Sarris (tsarris@ee.duth.gr) \\ Received: 12 June 2015 - Revised: 12 April 2016 - Accepted: 19 April 2016 - Published: 13 June 2016
}

\begin{abstract}
Ultra-low-frequency (ULF) pulsations are critical in radial diffusion processes of energetic particles, and the power spectral density (PSD) of these fluctuations is an integral part of the radial diffusion coefficients and of assimilative models of the radiation belts. Using simultaneous measurements from two Geostationary Operational Environmental Satellites (GOES) geosynchronous satellites, three satellites of the Time History of Events and Macroscale Interactions during Substorms (THEMIS) spacecraft constellation and the two Van Allen probes during a 10-day period of intense geomagnetic activity and ULF pulsations of October 2012, we calculate the PSDs of ULF pulsations at different $L$ shells. By following the time history of measurements at different $L$ it is shown that, during this time, ULF wave power is not enhanced uniformly throughout the magnetosphere but instead is mostly enhanced in the outer $L$ shells, close to the magnetopause, and to a lesser extent in the inner magnetosphere, closer to the plasmapause. Furthermore, by using phase differences between two GOES geosynchronous satellite pairs, we estimate the daily-averaged distribution of power at different azimuthal wave numbers. These results can have significant implications in better defining the effect of radial diffusion in the phase space density of energetic particles for different wave numbers or $L$ shell distributions of ULF power.
\end{abstract}

Keywords. Magnetospheric physics (energetic particles trapped; magnetospheric configuration and dynamics; storms and substorms)

\section{Introduction}

Ultra-low-frequency (ULF) fluctuations in the Earth's magnetosphere have frequencies in the millihertz $(\mathrm{mHz})$ range, within the drift frequency of energetic particles in the magnetosphere. For example, particles of energies $0.5,1.0$ and $5.0 \mathrm{MeV}$ have drift frequencies $\sim 1.0,1.7$ and $7.0 \mathrm{mHz}$, respectively, at geosynchronous orbit. As such, they have long been considered to play a key role in the acceleration of energetic particles through radial diffusion (Schulz and Lanzerotti, 1974; Brautigam and Albert, 2000; Li and Temerin, 2001). Radial diffusion is a process that violates the third adiabatic invariant of energetic particles through fluctuations and stochastic variations in the magnetic and electric fields in the magnetosphere, while conserving the first adiabatic invariant; thus the radial transport during a diffusive process results in the gain of energy for particles that diffuse inwards and the loss of energy for particles that diffuse outwards (Barker et al., 2005; Ukhorskiy et al., 2005; Elkington 2006; Degeling et al., 2008).

In radial diffusion models, the effects of ULF waves are described by the diffusion coefficient, $D_{\mathrm{LL}}$, which is separated into the electrostatic and the electromagnetic diffusion coefficient, $D_{\mathrm{LL}}^{\mathrm{E}}$ and $D_{\mathrm{LL}}^{\mathrm{M}}$, respectively. Several studies have provided estimates of the diffusion coefficients: Brautigam and Albert (2000) provided an analytic expression for $D_{\mathrm{LL}}^{\mathrm{E}}$ by assuming the electric field amplitude to be a linear function of $\mathrm{Kp}$, whereas for $D_{\mathrm{LL}}^{\mathrm{M}}$ they derived an analytic formula based on a combination of ground magnetometer measurements mapping to $L=4$ and spacecraft measurements at geosynchronous orbit. Brautigam et al. (2005) used CRESS electric field measurements to provide an analytic 
expression for $D_{\mathrm{LL}}^{\mathrm{E}}$ depending on $\mathrm{Kp}$ and $L$; Brautigam et al. (2005) showed that the electromagnetic diffusion coefficient by Brautigam and Albert (2000) is dominant over their corresponding electrostatic component. Ozeke et al. (2012) obtained an analytic expression for $D_{\mathrm{LL}}^{\mathrm{E}}$ by using ground magnetic field measurements at different $L$ shells which they mapped to the equatorial plane. They also obtained expressions for $D_{\mathrm{LL}}^{\mathrm{M}}$, using long-term Geostationary Operational Environmental Satellites (GOES) magnetic field measurements complemented by Active Magnetospheric Particle Tracer Explorers (AMPTE) measurements and showed that it is the electric field diffusion coefficient which dominates over the magnetic field diffusion coefficient across all $L$ shells spanning the outer radiation belt. Ozeke et al. (2014) presented simpler analytic expressions for ULF wave-derived radiation belt radial diffusion coefficients, as a function of $L$ and $\mathrm{Kp}$, which can easily be incorporated into global radiation belt transport models. They showed that the overall electric and magnetic diffusion coefficients are to a good approximation both independent of energy. Recently, Liu et al. (2016) used 7 years of Time History of Events and Macroscale Interactions during Substorms (THEMIS) electric field measurements to derive the diffusion coefficient due to electric field fluctuations and found significant differences between their model and the one by Ozeke et al. (2014); namely, they found a smaller $\mathrm{Kp}$ dependence and also an energy dependence, which is not included in the Ozeke et al. (2014) model.

In the following we focus on the radial diffusion effects of the compressional magnetic field component of ULF waves. The diffusion coefficient in this case is described by the following formulation:

$D_{\mathrm{LL}}^{\mathrm{B}, \mathrm{Sym}}=\frac{\mu^{2}}{8 q^{2} B_{\mathrm{E}}^{2} R_{\mathrm{E}}^{4}} \frac{L^{4}}{\gamma^{2}} \sum_{m} m^{2} P_{m}^{B}\left(m \omega_{\mathrm{d}}\right)$,

where $\mu, q, \gamma$ and $\omega_{\mathrm{d}}$ are the adiabatic invariant, charge, relativistic correction factor and drift frequency, respectively, of the particles under consideration; $B_{\mathrm{E}}$ is the Earth's magnetic field at the Earth surface; $m$ is the azimuthal wave number; and $P_{m}^{B}$ is the wave power spectral density (PSD) at $m$ times the particle drift frequency (Fei et al., 2006). In this expression marked in the shaded box are the parameters that are dependent on the ULF waves. Through this expression, the diffusion coefficient can be expressed as a function of $L$. This overcomes the need to estimate ULF wave power as a function of local time (azimuthal distribution) and only requires wave power to be evaluated as a function of $L$ shell on the equatorial plane (radial distribution).

In the following, we present an overview of some of the studies that have shown a dependence of ULF wave power on $L$ and of solar wind activity: it is known that there is a strong correlation between solar activity and ULF wave power (Engebretson et al., 1998), with the highest correlations occurring in the declining phase of the solar cycle when the radiation belts are most intense (Mann et al., 2004; Li et al., 2011). In particular, a number of studies have identified the solar wind as a key external source of ULF wave power (e.g., Mathie and Mann, 2001). Solar wind control of ULF waves has also been demonstrated by, e.g., Takahashi and Ukhorskiy (2007) using data from geosynchronous orbit. Mathie and Mann (2001) reported that ULF wave power increases with increasing $L$ shell and is typically an order of magnitude higher at $L=6.6$ than at $L=4$. They also demonstrated an exponential decay of $1-10 \mathrm{mHz}$ Pc5 wave power with decreasing $L$ shell, the decay rate increasing with solar wind speed, thus indicating a stronger dependence of pulsation power on solar wind speed at higher $L$ shells in the range $L=3.75-6.79$. Rae et al. (2012) extended the $L$ shell range in the study of Mathie and Mann (2001) to both lower and higher values and found that the ULF wave power continues to decrease toward lower $L$ shells, but at higher $L$ shells $(L \sim 8)$ the ULF powers also begin to decrease. Ozeke et al. (2014) used statistical GOES and THEMIS measurements to derive an analytic expression for power vs. $L$ for various geomagnetic activity levels, which can easily be incorporated into global radiation belt models. Recently, Dimitrakoudis et al. (2015) found that Kp is the best single parameter to specify the statistical ULF wave power driving radial diffusion. Moreover, two-parameter ULF wave power specifications using Dst as well as Kp provide a better statistical representation of storm-time radial diffusion than any single variable alone.

In this study we show results of compressional magnetic field PSD during a high-speed stream event, using measurements from the Van Allen probes, the THEMIS satellite constellation and GOES geosynchronous satellites. Furthermore, we use GOES geosynchronous measurements to estimate the distribution of power in the various wave numbers.

\section{Case study: storm of 8 October 2012}

Three consecutive geomagnetic storms occurred in the first half of October 2012: on 1, 8 and 13 October. The first storm, as observed by the Van Allen probes and reported by Baker et al. (2013), depleted the outer electron belt, and electron fluxes remained low and constant until the second storm. During the second storm, and in particular after 9 October 2012, the two RBSP satellites measured an intense relativistic electron enhancement event: electrons at all energies became enhanced, with lower-energy electrons being enhanced earlier and penetrating further inward ( $\mathrm{Li}$ et al., 2013). During the same event, the development of peaks in electron phase space density were observed (Reeves et al., 2013), which are considered evidence for local electron acceleration in the heart of the outer radiation belt. The third storm was characterized by a moderate drop of Dst to $-90 \mathrm{nT}$, on 13 October. The interval under study in this paper 
is the period from 7 to 11 October, which covers all phases of the second of the October 2012 storms.

\section{Measurements and methodology}

The Van Allen Probes mission (Mauk et al., 2012), initially named the Radiation Belt Storm Probes (RBSP) mission, was launched on 30 August 2012 and consists of two identical satellites, hereafter termed probes RBSP-A and RBSP-B, which orbit the Earth with an apogee of $\sim 5.7 R_{\mathrm{E}}$, an inclination of $10^{\circ}$, periods of $9 \mathrm{~h}$ and an orbital precession rate of $\sim 200^{\circ} \mathrm{yr}^{-1}$. In this study, measurements from the Electric and Magnetic Field Instrument Suite and Integrated Science (EMFISIS) are used. EMFISIS provides measurements of DC magnetic fields and a comprehensive set of wave electric and magnetic fields (Kletzing et al., 2013).

The THEMIS mission (Angelopoulos, 2008) originally consisted of five nearly identical satellites, hereafter termed probes TH-A through TH-E, which orbited the magnetosphere at different apogee; during the time of interest of this study, three of the satellites remain in the magnetosphere, while two have been moved into orbit near the Moon. The three remaining satellites are probes TH-A, TH-D and TH-E, orbiting at nearly identical orbits around Earth with apogees of $11.7 R_{\mathrm{E}}$, orbital periods of 1 day, inclinations ranging from 1 to $8^{\circ}$ and an orbital precession rate of $\sim 330^{\circ} \mathrm{yr}^{-1}$.

The Geostationary Operational Environmental Satellites provide continuous monitoring from a geosynchronous orbit. Most commonly there is one satellite over the east coast of the US at longitude $75^{\circ} \mathrm{W}$ (GOES-13 on October 2012) and one over the west coast at longitude $135^{\circ} \mathrm{W}$ (GOES-15); however during this period there are measurements available from a third satellite at longitude $100^{\circ} \mathrm{W}$ (GOES-14), which was in on-orbit storage and on its way to an ultimate longitude of $75^{\circ} \mathrm{W}$, moving east at a rate of $0.9^{\circ}$ per day. GOES satellite magnetic field measurements are available from the three-axis fluxgate magnetometers, which provide the magnetic field at a sampling period of $0.512 \mathrm{~s}$.

Magnetic field measurements from all the above platforms are rotated into a mean-field-aligned (MFA) coordinate system, which allows the magnetic field oscillations to be determined as toroidal (azimuthal), poloidal (radial) or compressional (parallel).

\section{Calculations of ULF wave power vs. $L$ shell}

We use simultaneous measurements of broadband ULF waves by the above measurement platforms (two Van Allen probes, three THEMIS and three GOES satellites) in order to reconstruct the $L$ dependence of ULF power spectral density in the radiation belts. The goal is to provide an estimate of the $L$ dependence of geomagnetic ULF waves in a format that can be used in estimates of radial diffusion at different phases of the storm.
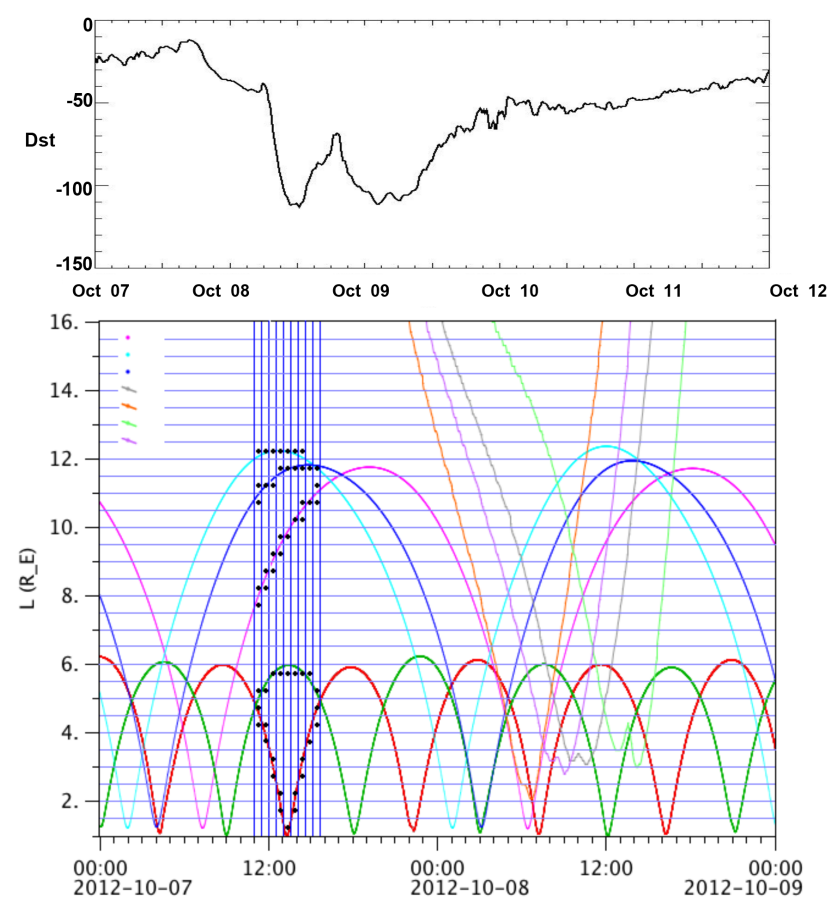

Figure 1. (a) Dst index from 7 to 12 October 2012 and (b) sample $L$ shell coverage of the Van Allen probes A and B (red and green lines, respectively) and the THEMIS probes A, D and E (purple, light blue and blue, respectively).

In Fig. 1 we show the $L$ shell of the Van Allen probes A and B (red and green lines, respectively), and of the THEMIS probes $\mathrm{A}, \mathrm{D}$ and $\mathrm{E}$ (purple, light blue and blue, respectively). As an example, in the same plot we show sample time bins (vertical blue lines) and $L$ shell bins (horizontal blue lines). Within any given time bin all the above satellites provide magnetic field measurements, together with GOES at geosynchronous orbit. The length of each time bin is the shortest possible that can provide ULF measurements in the Pc5 and Pc4 range with sufficient accuracy. A time bin of $1200 \mathrm{~s}$ was selected in this study. Similarly, the width of $L$ shell bins was selected so that, for the given time bin length, the satellites cross a minimum number of different $L$ shells. An $L$ shell bin of 0.25 was selected in this study.

Subsequently, after performing coordinate transformations from GSM coordinates to the mean-field-aligned coordinate system, we calculated the dynamic PSDs (power spectral densities as a function of time), which were subsequently averaged within each time bin. The corresponding $L$ shell is the average $L$ of each $s / c$ within each time bin.

The results are plotted in Fig. 2, where the power spectral density is plotted in color in a frequency-vs. $L$ plot, from 2 to $35 \mathrm{mHz}$ and $L=4$ to 12.5 , for 8 October 2012, 00:00 UT. In the first panel the instantaneous power is shown at the six locations of the spacecraft whose magnetic field measurements are used in this study; spacecraft names are written on the top of the panel, and arrows under the names indicate the 

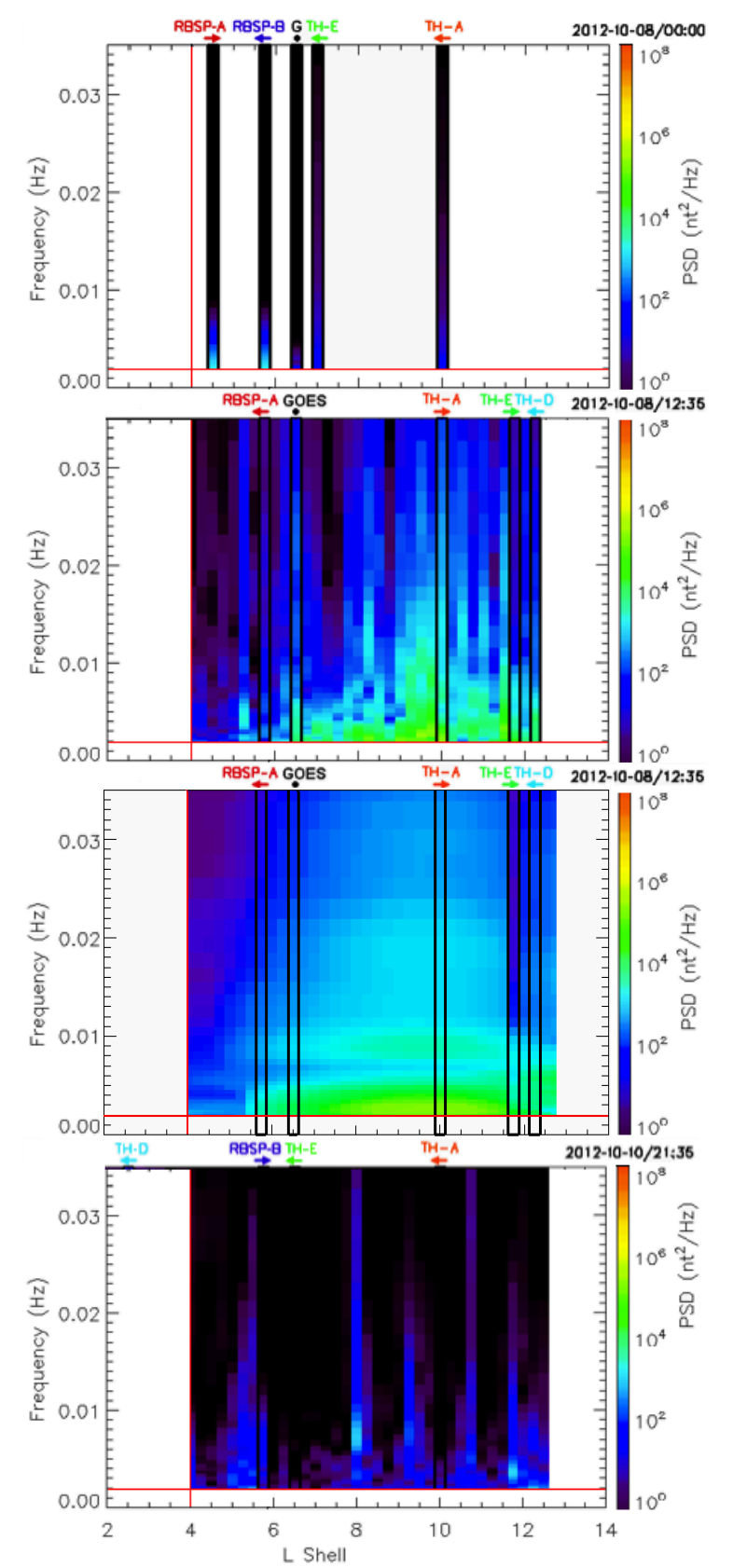

\section{Calculations of power vs. azimuthal wave number $m$}

In the following we use cross-spectral and cross-phase calculations between the time series of magnetic field measurements from azimuthally aligned satellites to get the fraction of total power in each wave number. The theoretical background for this technique has been discussed in greater detail in Sarris (2014). There it was described that, for example, between two measurement points that are separated in azimuth by $\Delta \varphi$, a wave number $m=0$ in the magnetosphere (corresponding to global oscillations) would be measured at the two points with $0^{\circ}$ phase difference. Also, pulsations of $m=1$ (hemispheric oscillations) would appear to have a $2 \pi$ modulation around the Earth and would thus appear to have a $\Delta \varphi$ phase difference between the two points. Similarly, $m=2$ pulsations would have a $4 \pi$ variation across all local times and would appear to have a phase difference of $2 \times \Delta \varphi$ between the two magnetometers; e.g., $m=3$ would correspond to $3 \times \Delta \varphi$. Two magnetometers on board two satellites separated in azimuth by $\Delta \varphi$ in the above example would thus be able to detect wave numbers up to $2 \pi / \Delta \varphi$, by classifying the detected phase differences between the observed oscillations in bins of width $\Delta \varphi$. As described in Sarris (2014), this technique is entwined with uncertainties - such as, among others, the $2 \pi$ ambiguity, local time vs. temporal variations, etc. - but can provide a much better estimate of the power per wave number than current assumptions, which attribute all power to a single wave number, in lack of better estimates.

For the calculation of phase differences between the two time series as a function of time and at all frequencies a crosswavelet transform (XWT) technique is used (e.g., Grinsted et al., 2004; Sarris et al., 2013; Sarris, 2014). The results of this calculation are wavelet and cross-wavelet power spectral 


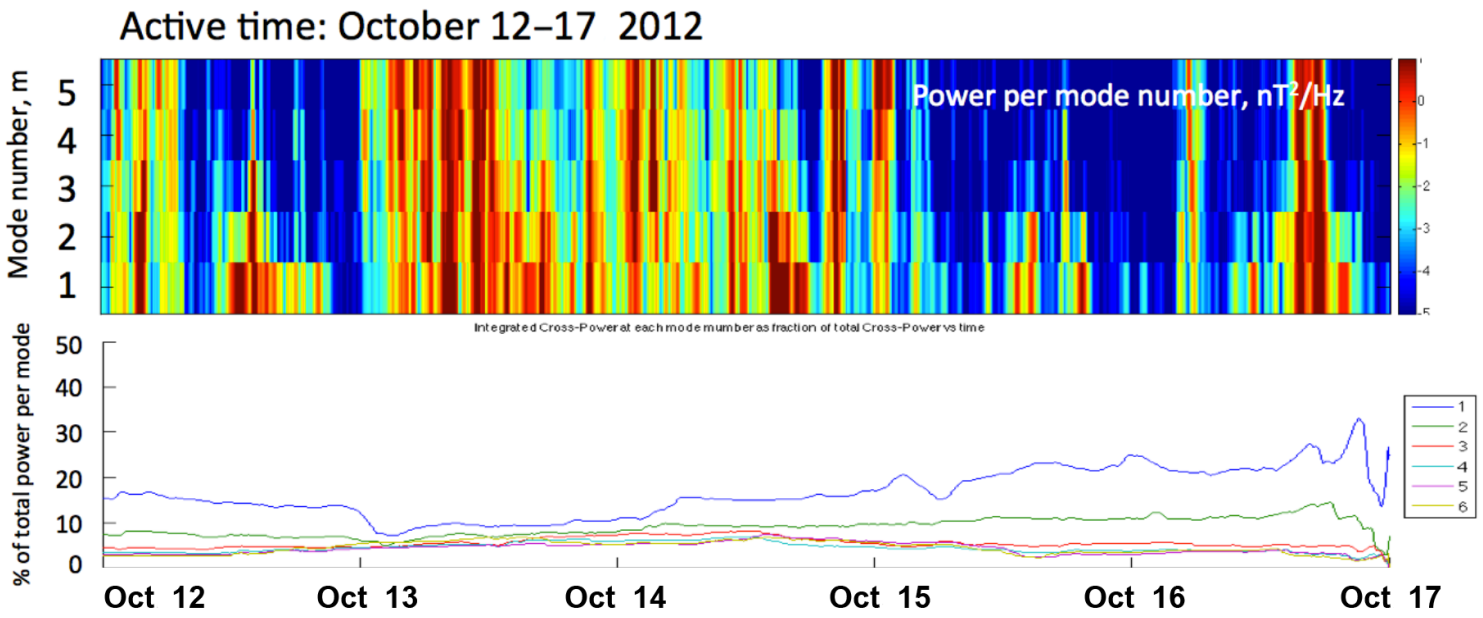

Figure 3. (a) Dst activity index; (b) calculations of the power per wave number using cross-spectrogram calculations between GOES-13 and GOES-15, at an azimuthal separation of $\sim 60^{\circ}$, as a function of time, from 12 to 17 October 2012; (c) fraction of power in one of the azimuthal wave numbers $m=1$ to 5 over total power in a time bin.

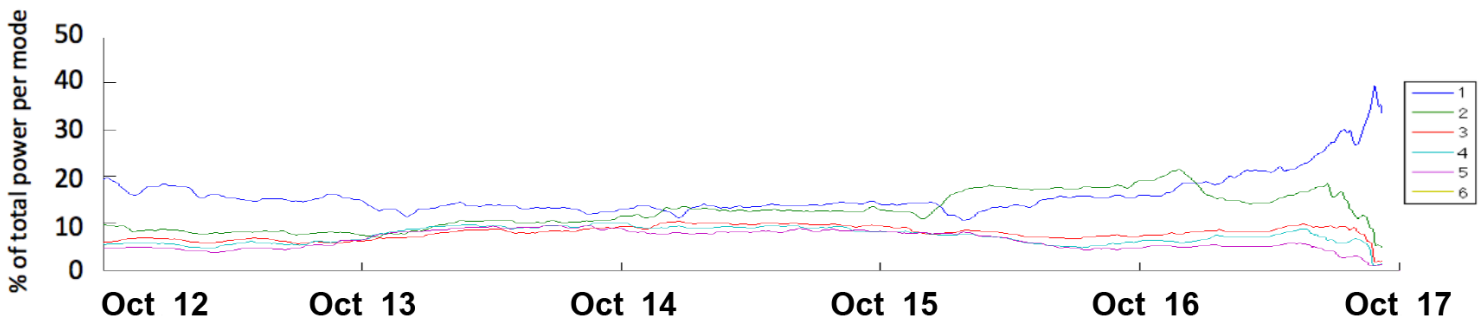

Figure 4. Calculations of the power per wave number using cross-spectrogram calculations between GOES-14 and GOES-15, at an azimuthal separation of $\sim 30^{\circ}$; the fractions of power in one of the wave numbers $m=1$ to 5 over total power in a time bin is given.

density (XWT-PSD) in units of $\mathrm{nT}^{2} \mathrm{~Hz}^{-1}$ and also the calculated phase difference as a function of frequency and time.

For the period in October 2012 we use phase difference calculations between the magnetometer measurements of GOES-13 and GOES-15 geosynchronous spacecraft, hereafter referred to as $\mathrm{G}_{13}$ and $\mathrm{G}_{15}$, which are azimuthally separated by $\sim 60^{\circ}$ during both events. The GOES satellites provide continuous monitoring from a geosynchronous orbit, with one satellite over the east coast of the US at longitude $75^{\circ} \mathrm{W}$ (GOES-13 in the two selected periods) and one over the west coast at longitude $135^{\circ} \mathrm{W}$ (GOES-15).

In the lower panel of Fig. 3 a moving average of the calculated $m$ is plotted; a sample window of 1 day was used for the moving average. This is due to the fact that the instantaneous calculation that can be provided through the pair of GOES spacecraft would estimate power over $m$ locally, at the instantaneous local time of the $s / c$; however, in order to provide a partitioning of ULF wave power in $m$ that can be used in estimates of the diffusion coefficient, the effects of ULF waves at a particular wave number need to be provided over all local times, instead of part of the particles' drift orbit around the Earth. We also note that megaelectronvolt electrons circulate the Earth in a fraction of a day, and so at each point of their orbit they interact with the local, instantaneous fields and wave number. Whether the averaged $m$ number can be used effectively in radial diffusion estimates instead of the localized and instantaneous $m$, and for which particle energies, needs to be resolved through particle-tracing simulations.

In the above calculation a critical assumption is used, namely that there is no spatial aliasing when calculating the phase differences of the waves between the two satellites. Thus, under this assumption, a $60^{\circ}$ separation can lead to the estimate of azimuthal wave numbers up to $m=6$ (following the argument that $\left.m=360^{\circ} / 60^{\circ}\right)$. However this calculation is not unambiguous, as it also contains the assumption that the wave numbers being observed are not multiples of $2 \pi$, in which case the azimuthal wave number could be $m, m+2 \pi$, $m+4 \pi$, etc., irrespective of how far apart the satellites are. In order to resolve this ambiguity, measurements at different azimuthal separations are needed, such as described in Sarris et al. (2013).

During the same time period under investigation, measurements from another geosynchronous satellite, GOES-14, were available. In order to identify if there are waves of a multiple of $2 \pi$, we have added calculations from the pair 
GOES-14 and GOES-15, which had approximately half the azimuthal separation. The results are shown in the Fig. 4. The fact that the fraction of power in each wave number is approximately the same for half the azimuthal separation provides an indication that the above assumption, which states the phase differences that are observed correspond to the lowest wave numbers, is valid and that indeed power in a wave number $m$ dominates over power at $m+2 \pi, m+4 \pi$, etc., minimizing the ambiguity of the results, at least in this case.

According to the technique used above, the azimuthal separation of $\sim 60^{\circ}$ between GOES-13 and GOES-15 allows for the calculation of wave numbers up to $m=6$. However we note that the power which this technique attributes to the higher wave numbers that can be calculated ( $m=6$ in this case) could be spatially aliased from other modes, as discussed in the technique outlined by Chisham and Mann (1999). We also note that the trend of the distribution of power from $m=1$ to progressively larger $m$ also includes critical information that can be used as an indicator for aliasing, which would arise from the existence of larger amounts of ULF wave power in wave numbers higher than what can be calculated. In particular, we note that power is monotonically decreasing from $m=1$ to $m=6$, with wave number $m=6$ getting, on average, less than $5 \%$ of the total power; we thus consider this aliasing to be insignificant. We do note, however, that an investigation of other periods beyond this event (not shown here) do show power in the last wave number, $m=6$, exceeding power in $m=4$ and 5, which could be an indication of enhanced power at higher wave numbers that can not be calculated due to the separation between the spacecraft; this could be used as an indication of the limitations of this methodology.

\section{Discussion}

The $L$ dependence of ULF wave power and also the distribution of power in each of the azimuthal wave numbers $m$ of ULF waves in the magnetosphere are integral parts of the characterization of the waves and are essential in the calculation of the rates of diffusion of energetic electrons and protons in the magnetosphere, as electrons and protons of drift frequency $\omega_{\mathrm{d}}$ have been shown to resonantly interact with ULF waves of frequency $\omega=m \omega_{\mathrm{d}}$. However, often in simulations of radial diffusion a simplifying assumption is made, namely that all ULF wave power can be attributed to waves of a single azimuthal wave number. In this study we show that a combination of spacecraft across different $L$ shell at a particular time can give an indication of the $L$ dependence during this time. Furthermore, the distribution of spacecraft across different local times can help provide an estimate of the distribution of power across different wave numbers. These results need to be further investigated through statistical studies, and empirical relationships need to be derived that can be used in models of radial diffusion in the radiation belts. A detailed statistical study involving GOES $s / c$ at closer distances would reveal the conditions for a monotonic decrease in ULF wave power with increasing $m$ to be observed, compared to cases when aliasing is observed, which would possibly also indicate the distribution of power to higher wave numbers. This information will lead to better understanding of the structure and propagation of ULF waves at different local times, as well as to a more accurate calculation of the diffusion rates that also takes into account a realistic azimuthal structure of broadband ULF waves.

\section{Data availability}

Data used in the above analyses are available at: http:// cdaweb.gsfc.nasa.gov/istp_public/ (Coordinated Data Analysis Web, CDAWeb).

Acknowledgements. We thank the Van Allen Probes Science Team and B. Mauk for Van Allen Probes data, the THEMIS Science Team and Vasilis Angelopoulos for THEMIS data, and NOAA and Howard Singer for GOES magnetometer data. This study was supported by NASA grants (THEMIS, NNX15AF56G and NNX12AG37G) and NSF grant ATM 0842388.

The topical editor, G. Balasis, thanks L. Ozeke and two anonymous referees for help in evaluating this paper.

\section{References}

Angelopoulos, V.: The THEMIS Mission, Sp. Sci. Rev., 141, 5-34, doi:10.1007/s11214-008-9336-1, 2008.

Baker, D. N., Kanekal, S. G., Hoxie, V. C., Henderson, M. G., Li, X., Spence, H. E., Elkington, S. R., Friedel, R. H. W., Goldstein, J., Hudson, M. K., Reeves, G. D., Thorne, R. M., Kletzing, C. A., and Claudepierre, S. G.: A long-lived relativistic electron storage ring embedded in Earth's outer Van Allen belt, Science, 340, 186-190, doi:10.1126/science.1233518, 2013.

Barker, A. B., Li, X., and Selesnick, R. S.: Modeling the radiation belt electrons with radial diffusion driven by the solar wind, Space Weather, 3, S10003, doi:10.1029/2004SW000118, 2005.

Brautigam, D. H. and Albert, J. M.: Radial diffusion analysis of outer radiation belt electrons during the 9 October 1990 magnetic storm, J. Geophys. Res., 105, 291-309, doi:10.1029/1999JA900344, 2000.

Brautigam, D. H., Ginet, G. P., Albert, J. M., Wygant, J. R., Rowland, D. E., Ling, A., and Bass, J.: CRRES electric field power spectra and radial diffusion coefficients, J. Geophys. Res., 110, A02214, doi:10.1029/2004JA010612, 2005.

Chisham, G. and Mann, I. R.: A Pc5 ULF wave with large azimuthal wavenumber observed within the morning sector plasmasphere by Sub-Auroral Magnetometer Network, J. Geophys. Res., 104, 14717-14727, doi:10.1029/1999JA900147, 1999.

Degeling, A. W., Ozeke, L. G., Rankin, R., Mann, I. R., and Kabin, $\mathrm{K}$.: Drift resonant generation of peaked relativistic electron distributions by Pc 5 ULF waves, J. Geophys. Res., 113, A02208, doi:10.1029/2007JA012411, 2008. 
Dimitrakoudis, S., Mann, I. R., Balasis, G., Papadimitriou, C., Anastasiadis, A., and Daglis, I. A.: Accurately specifying stormtime ULF wave radial diffusion in the radiation belts, Geophys. Res. Lett., 42, 5711-5718, doi:10.1002/2015GL064707, 2015.

Elkington, S. R.: A review of ULF interactions with radiation belt electrons, in: Magnetospheric ULF Waves: Synthesis and New Directions, edited by: Takahashi, K., Chi, P. J., Denton, R. E., and Lysak, R. L., AGU Geophysical Monograph, 169, AGU, Washington, DC, 2006.

Fei, Y., Chan, A. A., Elkington, S. R., and Wiltberger, M. J.: Radial diffusion and MHD particle simulations of relativistic electron transport by ULF waves in the September 1998 storm, J. Geophys. Res., 111, A12209, doi:10.1029/2005JA011211, 2006.

Grinsted, A., Moore, J. C., and Jevrejeva, S.: Application of the cross wavelet transform and wavelet coherence to geophysical time series, Nonlin. Processes Geophys., 11, 561-566, doi:10.5194/npg-11-561-2004, 2004.

Kletzing, C. A., Kurth, W. S., Acuna, M., McDowall, R. J., Torbert, R. B., Averkamp, T., Bodet, D., Bounds, S. R., Chutter, M., Connerney, J., Crawford, D., Dolan, J. S., Dvorsky, R., Hospodarsky, G. B., Howard, J., Jordanova, V., Johnson, R. A., Kirchner, D. L., Mokrzycki, B., Needell, G., Odom, J., Mark, D., Pfaff, R., Phillips, J. R., Piker, C. W., Remington, S. L., Rowland, D., Santolik, O., Schnurr, R., Sheppard, D., Smith, C. W., Thorne, R. M., and Tyler, J.: The Electric and Magnetic Field Instrument Suite and Integrated Science (EMFISIS) on RBSP, Space Sci. Rev., 179, 127-181, doi:10.1007/s11214-013-9993-6, 2013.

$\mathrm{Li}, \mathrm{X}$. and Temerin, M.: The electron radiation belt, Space Sci. Rev., 95, 569-580, doi:10.1023/A:1005221108016, 2001.

Li, X., Temerin, M., Baker, D. N., and Reeves, G. D.: Behavior of $\mathrm{MeV}$ electrons at geosynchronous orbit during last two solar cycles, J. Geophys. Res., 116, A11207, doi:10.1029/2011JA016934, 2011.

Li, X., Schiller, Q., Blum, L., Califf, S., Zhao, H., Tu, W., Turner, D. L., Gerhardt, D., Palo, S., Kanekal, S., Baker, D. N., Fennell, J., Blake, J. B., Looper, M., Reeves, G. D., and Spence, H.: First results from CSSWE CubeSat: Characteristics of relativistic electrons in the near-Earth environment during the October 2012 magnetic storms, J. Geophys.Res.-Space, 118, 6489-6499, doi:10.1002/2013JA019342, 2013.

Liu, W., Tu, W., Li, X., Sarris, T., Khotyaintsev, Y., Fu, H., Zhang, H., and Shi, Q.: On the calculation of electric diffusion coefficient of radiation belt electrons with in situ electric field measurements by THEMIS, Geophys. Res. Lett., 43, 1023-1030, doi:10.1002/2015GL067398, 2016.

Mann, I. R., O’Brien, T. P., and Milling, D. K.: Correlations between ULF wave power, solar wind speed, and relativistic electron flux in the magnetosphere: solar cycle dependence, J. Atmos. Sol.-Terr. Phys., 66, 187-198, doi:10.1016/j.jastp.2003.10.002, 2004.
Mathie, R. A. and Mann, I. R.: On the solar wind control of Pc5 ULF pulsation power at mid-latitudes: Implications for $\mathrm{MeV}$ electron acceleration in the outer radiation belt, J. Geophys. Res., 106, 29783-2979, 2001.

Mauk, B. H., Fox, N. J., Kanekal, S. G., Kessel, R. L., Sibeck, D. G., and Ukhorskiy, A.: Science Objectives and Rationale for the Radiation Belt Storm Probes Mission, Space Sci. Rev., 179, $3-$ 27, doi:10.1007/s11214-012-9908-y, 2012.

Ozeke, L. G., Mann, I. R., Murphy, K. R., Rae, I. J., Milling, D. K., Elkington, S. R., Chan, A. A., and Singer, H. J.: ULF wave derived radiation belt radial diffusion coefficients, J. Geophys. Res., 117, A04222, doi:10.1029/2011JA017463, 2012.

Ozeke, L. G., Mann, I. R., Murphy, K. R., Jonathan Rae, I., and Milling, D. K.: Analytic expressions for ULF wave radiation belt radial diffusion coefficients, J. Geophys. Res.-Space, 119, 15871605, doi:10.1002/2013JA019204, 2014.

Rae, J., Mann, I. R., Murphy, K. R., Ozeke, L. G., Milling, D. K., Chan, A. A., Elkington, S. R., and Honary, F.: Ground-based magnetometer determination of in situ Pc4-5 ULF electric field wave spectra as a function of solar wind speed, J. Geophys. Res.Space, 117, A04221, doi:10.1029/2011JA017335, 2012.

Reeves, G. D., Spence, H. E., Henderson, M. G., Morley, S. K., Friedel, R. H., Funsten, H. O., Baker, D. N., Kanekal, S. G., Blake, J. B., Fennell, J. F., Claudepierre, S. G., Thorne, R. M., Turner, D. L., Kletzing, C. A., Kurth, W. S., Larsen, B. A., and Niehof, J. T.: Electron acceleration in the heart of the Van Allen radiation belts, Science, 341, 999-994, doi:10.1126/science.1237743, 2013.

Sarris, T. E.: Estimates of the power per mode number of broadband ULF waves at geosynchronous orbit, J. Geophys. Res.-Space, 119, 5539-5550, doi:10.1002/2013JA019238, 2014.

Sarris, T. E., Li, X., Liu, W., Argyriadis, E., Boudouridis, A., and Ergun, R.: Mode number calculations of ULF fieldline resonances using ground magnetometers and THEMIS measurements, J. Geophys. Res.-Space, 118, 6986-6997, doi:10.1002/2012JA018307, 2013.

Schulz, M. and L. J. Lanzerotti: Particle Diffusion in the Radiation Belts, Springer-Verlag, New York, 1974.

Takahashi, K. and Ukhorskiy, A. Y.: Solar wind control of Pc5 pulsation power at geosynchronous orbit, J. Geophys. Res., 112, A11205, doi:10.1029/2007JA012483, 2007.

Ukhorskiy, A. Y., Takahashi, K., Anderson, B. J., and Korth, H.: The impact of inner magnetospheric ULF waves on the outer radiation belt electrons, J. Geophys. Res., 110, A10202, doi:10.1029/2005JA011017, 2005. 